\title{
Brain Imaging Increases Our Understanding of Developmental Coordination Disorder: a Review of Literature and Future Directions
}

\author{
Meisan Brown-Lum ${ }^{1}$ • Jill G. Zwicker ${ }^{1}$
}

Published online: 26 March 2015

(C) Springer International Publishing Switzerland 2015

\begin{abstract}
Developmental coordination disorder (DCD) is a neuromotor disorder of unknown etiology characterized by poor motor skills that significantly interferes with a child's ability to perform everyday activities and affects their psychosocial well-being. Little is known about the neural mechanisms underlying motor impairment, making it difficult to understand why children with DCD struggle to learn motor skills and what the best intervention would be to optimize function. With the advent of advanced neuroimaging techniques, several MRI studies have been conducted to tackle this important issue. Findings from these studies suggest that children with DCD activate different regions of the brain during functional tasks and show differences in white matter microstructure compared to typically developing children. The emerging neuroimaging data will help clarify the possible underlying mechanisms in relation to impaired motor function at the behavioral level in children with DCD.
\end{abstract}

Keywords Developmental coordination disorder · DCD . Neuroimaging $\cdot$ fMRI $\cdot$ DTI $\cdot$ EEG

This article is part of the Topical Collection on Disorders of Motor

Jill G. Zwicker

jill.zwicker@ubc.ca

Meisan Brown-Lum

mbrownlum@alumni.ubc.ca

1 Department of Occupational Science and Occupational Therapy, Faculty of Medicine, The University of British Columbia, K3-180 4480 Oak Street, Vancouver, BC, Canada V6H 3V4

\section{Introduction}

Affecting 5-6\% of school-age children, developmental coordination disorder (DCD) is a neurodevelopmental disorder characterized by motor impairment that significantly interferes with a child's activities of daily living [1]. DCD is heterogeneous, where the core condition is difficulty with fine and/or gross motor skills [2,3]. Motor performance in children with DCD is slower, less accurate, and more varied than their peers, and upon assessment, children with DCD score below that expected for their age and intelligence. These motor impairments significantly impact daily life, and secondary psychosocial difficulties often develop, such as anxiety, depression, and poor self-esteem [4]. Children with DCD do not simply outgrow the motor impairment. Without intervention, it is estimated that nearly $75 \%$ of children with DCD continue to have difficulties as adults [2].

Behavioral work has contributed significant knowledge about the basis of DCD and has largely directed the recent neuroimaging studies [5•]. The underlying assumption driving the neuroimaging research is that impaired motor function is the result of atypical brain development. Compared to what is known about other developmental disorders, such as autism and attention-deficit hyperactivity disorder (ADHD), little is known of the neural basis of DCD [6]. This makes it difficult to understand why children with DCD struggle to learn motor skills and what the best intervention would be to optimize function. Neuroimaging research provides an objective marker into the neurophysiological basis of DCD [7]. In the same way that neuroimaging studies have informed intervention for cognitive skill learning such as reading $[8,9]$, the brain differences found in children with DCD compared to typically developing peers may increase our understanding of neural correlates associated with DCD and subsequently improve diagnosis and optimize intervention strategies [10]. This review 
will focus on neuroimaging techniques and summarize current knowledge gained from brain imaging studies on motor impairment in children with DCD. We conclude with a discussion on limitations of this work and future directions for research and practice.

\section{Methods}

For this review paper, we searched EMBASE, PubMed, and PsycInfo databases from January 1, 2000, to December 1, 2014. Our search included the keywords of "developmental coordination disorder" and "magnetic resonance imaging." Because the first search yielded only a few studies, we did a secondary search using the following keywords: Movement Assessment Battery for Children, neuromotor assessment, and neuroimaging. Studies were included if the operational definition of DCD diagnosis met DSM-IV or DSM-V criteria, if a standardized motor assessment such as the Movement Assessment Battery for Children (MABC) [11] was used, motor outcomes using imaging technology, and participant age of 5 years or older. According to the European Academy for Childhood Disability (EACD) guidelines for DCD, 5 years is the minimum age that DCD can be diagnosed [10]. Case reports and pilot studies were also included. Studies that included severe neurological conditions, such as cerebral palsy and autism, were excluded. In total, the search results yielded 22 studies, which included seven functional magnetic resonance imaging (fMRI) studies, two diffusion tensor imaging (DTI) studies, six electroencephalogram (EEG) studies, and one case study using single-photon emission computed tomography (SPECT). All children in the studies met diagnostic criteria for DCD and used the MABC-2 to assess motor impairment. MABC-2 cutoff scores ranged from below the 16th percentile to below the 5 th percentile. All of the studies were small ranging from $N=7$ to $N=24$ with age-matched controls between the ages of 6 to 17 years (see Table 1 for a summary of the research findings).

\section{Brain Imaging Techniques to Study Motor Impairment in Children with DCD}

Since 2006, there have been advances in the application of neuroimaging technology to characterize brain development in children with DCD. Advanced imaging techniques such as fMRI, EEG, and DTI have been applied to characterize pathways and regions of activation that are involved in cortical sensorimotor processing. All of these modalities yield different levels of details about the brain that can help complement each other to build a better picture of the neurodevelopmental trajectory of children with DCD.

\section{Functional Magnetic Resonance Imaging}

fMRI is an indirect measure of neural activity that measures local blood flow by detecting changes in blood oxygenation (BOLD) and metabolic demands of active neurons. In response to neural activity, an increase in oxygenated hemoglobin flows into capillaries with a corresponding decrease in the amount of deoxygenated hemoglobin. This change in the ratio of oxygenated and deoxygenated hemoglobin causes susceptibility in the magnetic field that is measured using fMRI pulse sequences and can be visualized as an increase in image intensity [12].

To date, a handful of studies have applied fMRI to examine differences in brain function in children with DCD. These studies are summarized in Table 1. Building on behavioral research [13-17] showing differences in attention in children with and without DCD, Querne et al. in 2008 was the first to apply fMRI to test a theoretical model of response inhibition during a go-no-go task in children with DCD [18••]. They reported that children with DCD exhibited abnormal brain hemispheric specialization when performing a go-no-go task. Connectivity analysis revealed that children with DCD engage the same cerebral regions but in different ways than typically developing children. Children with DCD had lower activation in the dorsolateral prefrontal cortex (DLPFC) compared to the control group. These findings suggest that hypoactivation in the attentional network may impact the underlying mechanisms involved with disruption in motor planning observed in children with DCD. Further, in a recent fMRI study, Debrabant et al. investigated differences in predictive motor timing in a visually guided motor task. They reported hypoactivation in the right DLPFC, left posterior cerebellum, and right tempero-parietal junction in children with DCD. These findings suggest that children with DCD require extra processing efforts to perform visually guided predictive motor tasks compared to their TD peers [19].

To investigate brain function during a motor control task, Kashiwagi et al. in 2009, was the first to investigate the neural correlates of children with DCD during visuomotor tasks. The purpose of the study was to detect underlying motor control deficits in children with DCD. During tracking of a horizontally moving target, children with DCD demonstrated less activation in the left superior and inferior parietal lobules compared to TD children. These findings support the neurocognitive literature which suggests that the underlying mechanism of DCD may be associated with dysfunction in motor control involving the parietal lobe [20]. Using a different task that required greater demands for continued processing of visuospatial information, Zwicker et al. in 2010 reported greater, not lower, brain activation in the frontal, parietal, and temporal brain regions during a trail-tracing task. These findings suggest that children with DCD needed to recruit more attentional resources when first learning a motor task [21]. In 2011, Zwicker et al. was the first study to examine 


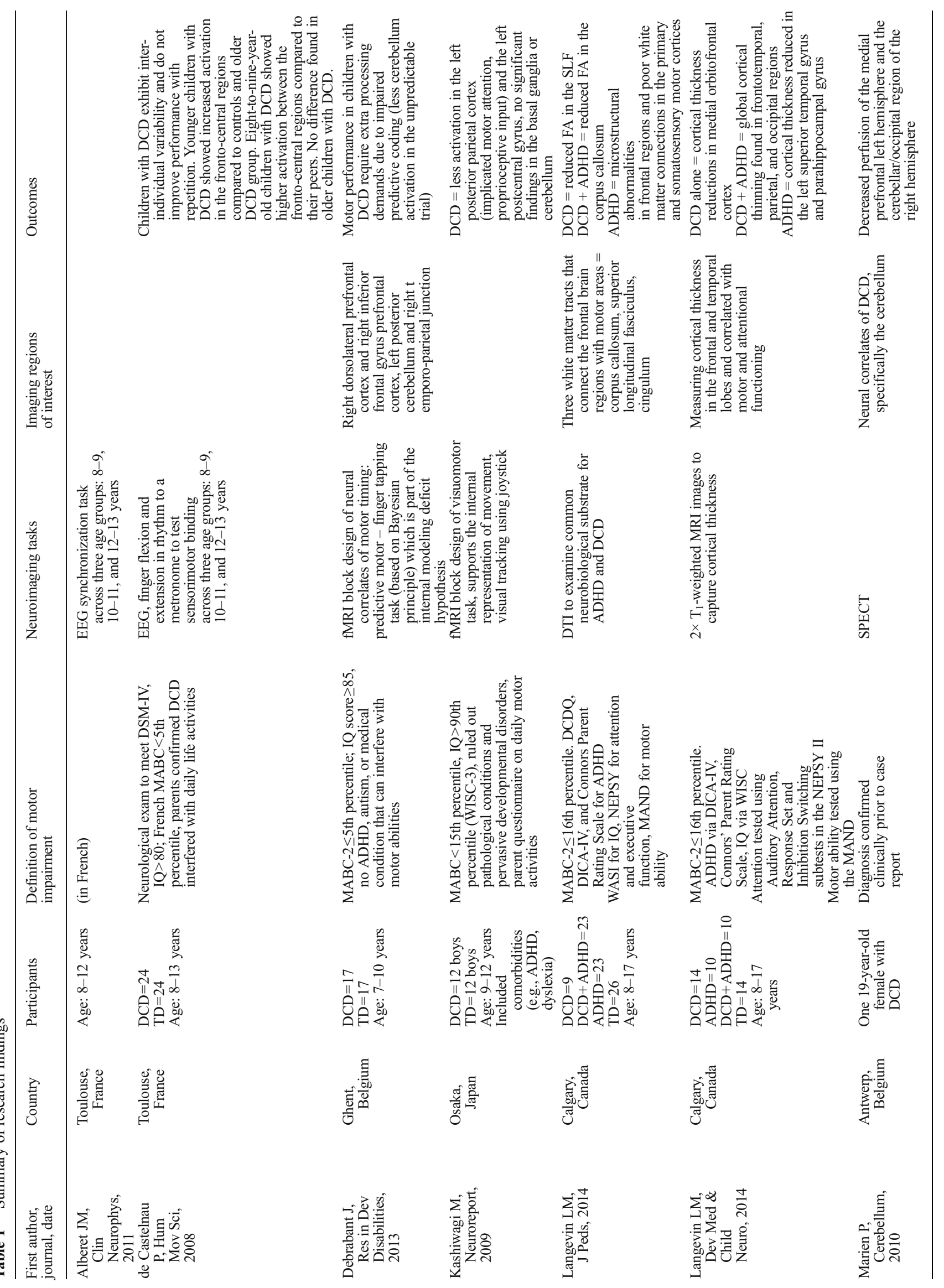




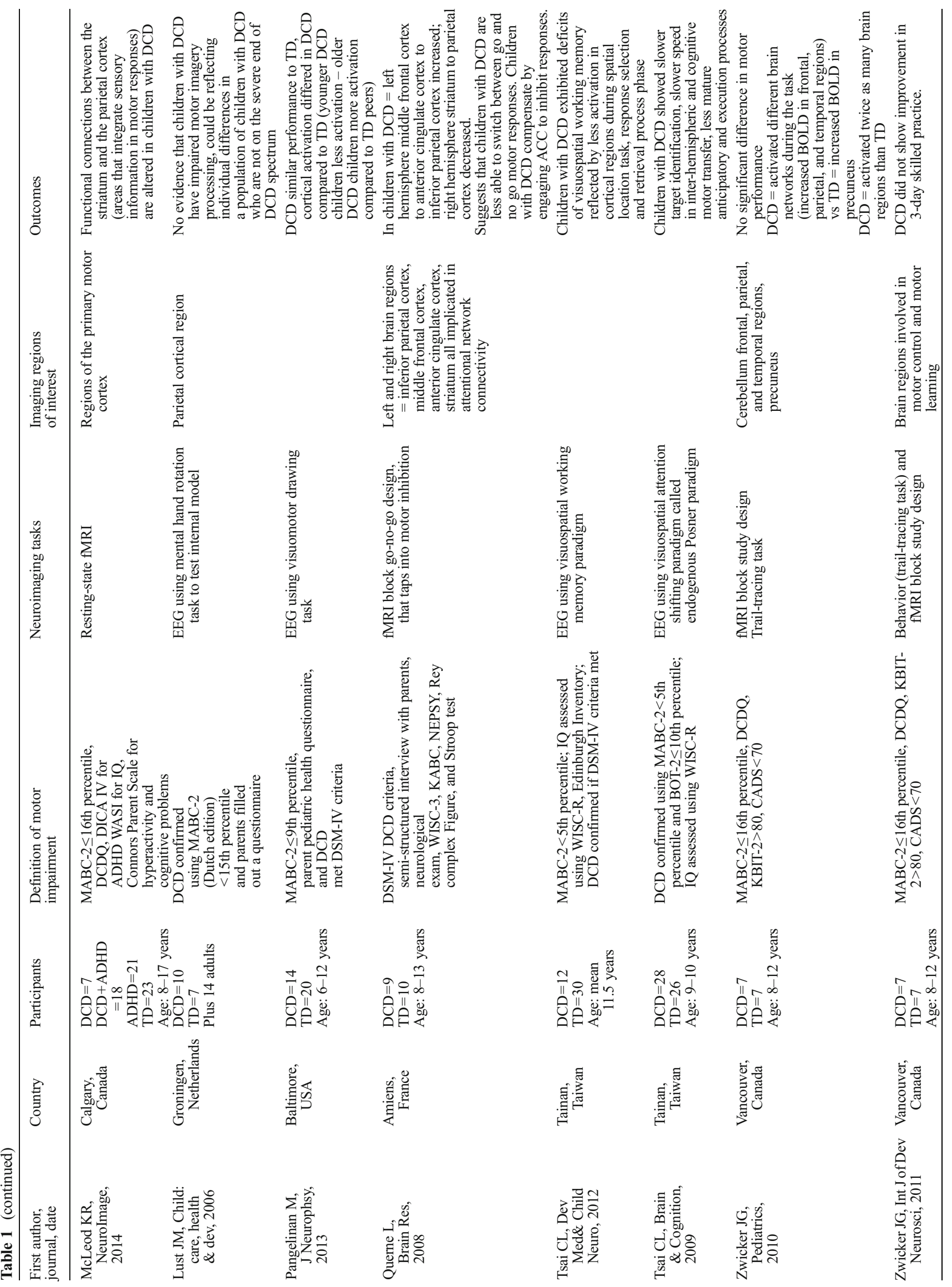




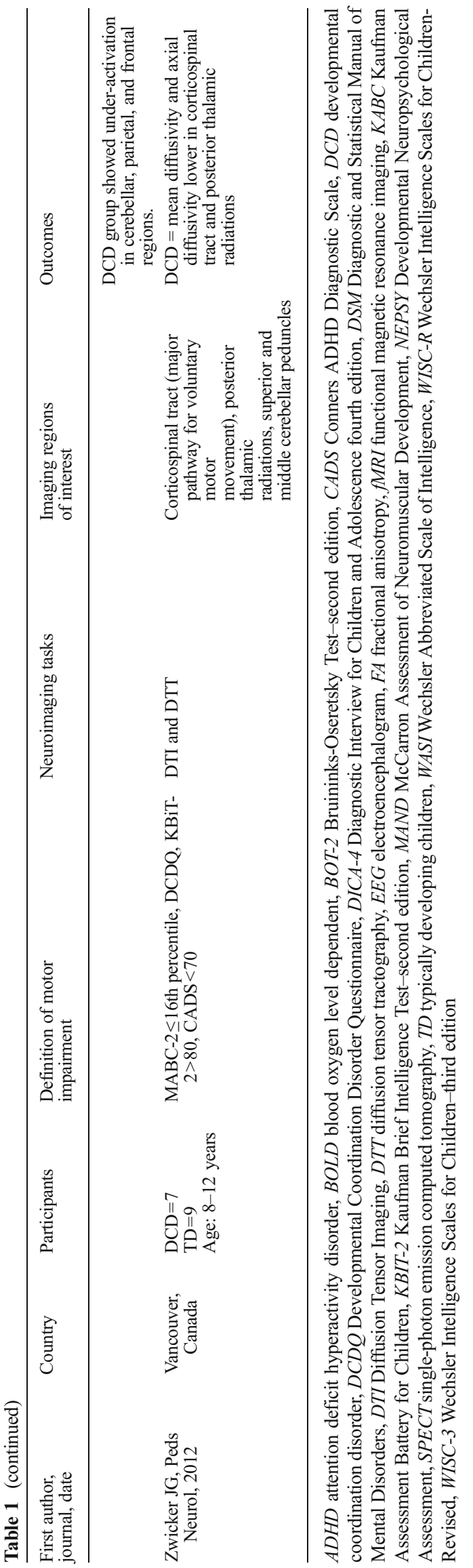

changes in the patterns of brain activation that were associated with skilled motor practice in children with DCD. The authors from this study concluded that children with DCD demonstrated a different pattern of activation from typically developing peers in the DLPFC, inferior parietal lobule, and the cerebellum $[22 \bullet \bullet]$. The DLPFC is associated with the initial stages of explicit motor learning and attentional control- these findings are consistent with the study of Querne et al. in 2008. Findings from Zwicker et al. in 2011 suggest possible inefficiencies between motor and sensory pathways, and the areas of activation implicated in DCD suggest that cerebellofrontal and cerebello-parietal pathways may be affected, which is supported by years of behavioral studies (see recent systematic review by Adams et al. [23]). This hypothesis needs to be confirmed with functional connectivity imaging analyses. DTI may also be helpful to determine if these specific networks are implicated in DCD.

A major limitation to interpreting these fMRI studies is that fMRI is not a direct measure of neuronal activity; it is an indirect measure of brain activity by detecting changes in blood flow and metabolic demands of active neurons. Further, fMRI studies use different tasks in the study design and that there are also limitations to using fMRI in the pediatric population as it requires children to keep still for long periods of time. Despite these limitations, functional MRI is critical to help characterize differences in patterns of brain activation in children with DCD as compared to controls. Larger studies that replicate these findings using the same cutoff scores to assess DCD and that use the same motor tasks would help build on the significance of these findings. Structural MRI will complement the functional data by providing morphological and connectivity differences in the brains of children with and without DCD.

\section{Resting-State Functional Magnetic Resonance Imaging}

Resting-state fMRI is a technique that identifies localized low frequency BOLD signals that are correlated when the brain is at rest [24]. Brain regions that are functionally linked in resting-state networks are anatomically connected [25]. Resting-state networks have been established in typically developing children [26, 27] and atypical patterns in neurodevelopment disorders such as ADHD and autism spectrum disorder [28, 29]. McLeod et al. looked at resting-state fMRI and reported for the first time, reductions in functional connectivity in children with DCD and/or ADHD in a number of brain regions involved in motor circuitry (e.g., primary motor cortex, inferior frontal gyri, the striatum, angular gyri). These findings suggest that overlapping patterns of brain activity may underlie motor and attention problems [30]. More studies are needed to investigate the functionally linked brain regions in children with motor impairment and the relationship of DCD with comorbid conditions. 


\section{Electroencephalography}

EEG is the oldest functional neuroimaging technique and measures electrical activity of neurons through the placement of electrodes on the scalp. EEG measures the current flow emitted by the synaptic interchanges produced by the dendrites of pyramidal neurons. EEG permits the study of neuronal events occurring at the millisecond level. Studies have established that EEG can be applied to visualize electrical activity directly in various structures of the brain and identify impaired brain development [31]. This technique is appealing as it is a more feasible and less invasive protocol for children than MRI.

A handful of studies have correlated EEG patterns of cortical activation with motor function in children with DCD. Brain regions of interest were the frontal lobe, parietal regions, and lateralization of the left and right hemispheres. In general, these studies have reported that compared with typically developing children, children with DCD performed similarly but exhibited different patterns of cortical activation $[32,33 \cdot, 34$, $\left.35 \cdot, 36^{\circ}\right]$ and suggest that children with DCD have deficits in visuospatial working memory [34, 35•]. For example, during a visuospatial working memory task, Tsai et al. in 2012 used EEG data to investigate cortical activity in children with and without DCD. They reported that children with DCD exhibited a retrieval deficit for spatial information, suggesting that children with DCD allocate fewer neural resources and less effort to stimulus evaluation and response selection and engage in less neural processing during the retrieval phase. EEG studies can contribute to the investigation of cortical development of DCD, and thus, more EEG studies are needed to build on the current findings.

\section{Diffusion Tensor Imaging}

DTI provides exquisite details about brain white matter tissue architecture in both normal and impaired states that one cannot see using conventional MRI. Currently, DTI is the only approach available to track brain white matter fibers noninvasively. Using DTI, diffusion data provide information on maturation and pruning of white matter fibers [37]. DTI measures the amount of water molecule displacement (mean diffusivity) and relative direction (fractional anisotropy or FA) of water diffusion in axons. Mean diffusivity decreases and FA increases with white matter maturation and greater microstructural integrity. Other diffusion parameters include axial diffusivity (water diffusion along the length of axons) and radial diffusivity (water diffusion perpendicular to axons). DTIderived measures are influenced by factors such as the amount of myelin and coherence in the organization of axonal tracts [38]. Increases in these measures reflect better white matter structural support for efficient and rapid transmission of information among different brain regions, supporting the development of well-functioning and synchronized neural pathways.

The importance of studying white matter architecture in children with DCD is crucial to understand impaired neurodevelopment and motor outcome, with DTI now an emerging field. In a pilot study, Zwicker in 2012 was the first to show that lower axial diffusivity of the corticospinal tract and the posterior thalamic radiations were highly correlated with motor outcomes in children with DCD compared to typically developing children; higher axial diffusivity was significantly correlated with higher motor scores [39••]. Further, Langevine et al. in 2014 found reduced FA in regions of the corpus callosum underlying the parietal cortex as well as the left superior longitudinal fasciculus. They also reported abnormalities in the parietal regions of the corpus callosum that form connections to both primary and somatosensory motor areas $[40 \bullet$ ]. Their findings also suggest that DCD and ADHD share overlapping etiology.

The issue of comorbidities is important because it can impact the research findings and interpretability. It is common knowledge that DCD can co-occur with other developmental disorders [6]. Due to the overlapping nature of developmental disorders, behavioral researchers have suggested that DCD may reflect a general developmental disorder of brain function, so called atypical brain development [41-43]. Children with DCD may have one or more disorders (e.g., ADHD), and depending on the extent of disruption to brain development, DCD may result from different neural mechanisms [44]. Therefore, it is important to recognize and perhaps differentiate DCD from other comorbidities when studying its neural correlates as this may help identify particular subtypes, based on different constellations of brain dysfunction.

\section{Structural Magnetic Resonance Imaging}

Whole brain analyses that use methods such as voxel-based morphometry derive gray matter volume, density, and thickness measurements, which reflect changes in the number of neuronal cell bodies, dendrites, and synaptic density [44]. A study by Langevin and colleagues looked at cortical thickness in a large sample of children with combined motor and attentional deficits $[45 \cdot \bullet]$ and reported cortical thinning in the frontal, parietal, and temporal lobes; these metrics were also correlated with aspects of motor and attentional functioning. There was distinct global pattern of cortical thickness decrease in children with DCD and comorbid ADHD. More studies are needed to investigate the neural indices of the structural brain of children with DCD.

\section{Single-Photon Emission Computed Tomography}

One case study using SPECT by Mariën et al. in 2010 reported a disruption of the cerebello-cerebral network that is involved 
in executing planned actions, visuospatial cognition, and affective regulation [46]. These findings build on earlier behavioral studies $[5 \cdot 23]$ and suggest that the cerebellum is implicated in the pathophysiological mechanisms of DCD and corroborate Zwicker's fMRI results [22••].

\section{Discussion}

This review of literature suggests that children with DCD show different patterns of neural activation during motor tasks compared to typically developing children. Further, DTI studies report preliminary evidence to suggest that the white matter architecture differs in children with DCD compared to typically developing children. These findings support the hypothesis that DCD is the result of atypical brain development and show unequivocally that children with DCD are neurobiologically different than their typically developing peers, lending credence to the legitimacy of DCD as a neurodevelopmental disorder. Findings from these and future brain imaging studies can inform our understanding of DCD in three important ways: (1) increasing our theoretical understanding of the disorder; (2) examining predictors of DCD, which may inform prevention strategies; and (3) investigating effectiveness and neuroplastic change associated with rehabilitation interventions for children with DCD.

\section{Brain Imaging Can Inform Our Theoretical Understanding of DCD}

The cerebellum has long been hypothesized to be involved in DCD $[6,47]$. It is especially vulnerable in developmental disorders because it develops relatively later and slower than most other brain regions [15]. In addition, there are two possible mechanisms that suggest that the cerebellum is involved in DCD, the automatization deficit hypothesis [48, 49], and the internal modeling deficit hypothesis $[6,23,50-52]$. The automatization deficit hypothesis suggests that children with DCD, like those with dyslexia, may have difficulty making motor skills automatic. Further neuroimaging studies comparing single-task versus a dual-task performance are needed to confirm this hypothesis. The internal modeling deficit hypothesis suggests that successful motor control is thought to result from an internal model involving the cerebellum that accurately predicts the sensory consequences of motor command [50].

Recent neuroimaging studies support the hypothesis from behavioral studies that the cerebellum and/or its networks are involved in DCD [6]. For example, a couple of studies reported that children with DCD exhibited lower activation in the DLPFC and the left posterior cerebellum [30, 53]. Zwicker et al. measured brain activation while children with and without DCD performed a trail-tracing task [22••]. Behaviorally, both groups performed similarly; however, BOLD analysis revealed that children with DCD had less activation in the right cerebellar crus I, left cerebellar lobule IV and IX, bilateral inferior parietal lobules, and right middle frontal gyrus compared to typically developing children. Despite the small sample size, this is the first study to support the possible relationship between cerebellar under-recruitment and poor motor performance in children with DCD. Further, in a single case study, structural MRI confirmed the cerebellar involvement in a 19 year-old with DCD, indicating functional disruption of the cerebellum [46]. Future brain imaging studies using motor learning tasks will be crucial to further our understanding of the role of the cerebellum in DCD.

The frontal-parietal connections are involved in serial processing of sensory motor networks (such as the visuospatial processes) and theories of motor skill learning. Results of a meta-analysis of the information processing deficits associated with DCD showed that children with DCD have significantly poorer visual spatial processing than healthy controls [43]. This suggests that the parietal region may be implicated in DCD given its role in processing visual spatial information $[6,51,52,54-56]$. To investigate parietal dysfunction in DCD, Zwicker et al. reported under-activation in the frontalparietal and frontal cerebellar networks when children with and without DCD performed a trail-tracing task [22••]. In another study using joystick tracing, Kashiwagi et al. reported lower brain activation in the left posterior parietal cortex and postcentral gyrus [20]. Abnormal functional connectivity was also reported in the parietal-related networks in DCD [18••, $19,45 \cdot \bullet$. These studies suggest that the suboptimal parietal function may be another neural correlate of DCD.

\section{Brain Imaging May Inform Ways to Prevent DCD in High-Risk Populations}

In a systematic review, Edwards et al. in 2011 reported that children born very preterm are at much higher risk compared to children born full-term to develop DCD [57]. The authors further highlighted the value in determining the mechanisms underlying DCD in the preterm population as this would help inform early identification and intervention. In particular, white matter injury has been hypothesized to account for motor impairment in children born preterm [58•]. More specifically, impaired maturation of the oligodendrocyte lineage and/ or myelination in white matter tracts associated with motor development, such as the hippocampus [59], corticospinal tract [6], cerebellum [60], and corpus callosum [61] have been implicated in motor impairments consistent with DCD. Further, reduced FA values have been reported to be strongly associated with motor impairment and DCD in very preterm children at school age $[62 \bullet, 63]$.

Zwicker et al. has investigated the association of ante-natal, peri-natal, and post-natal predictors of DCD on the development of the corticospinal tract, the main voluntary motor 
pathway [64], and reported that higher illness severity in the first days of life and use of painful procedures were associated with slower maturation of the corticospinal tract. Interestingly, this dysmaturation was related to lower radial diffusivity (water diffusion perpendicular to axons), suggesting that myelin and the cells that produce myelin are affected. In contrast, Zwicker et al. in 2012 found that lower axial diffusivity (water diffusion along the length of axons) was related to poorer motor skills in a term-born cohort of children with DCD [38]. Taken together, these studies suggest that different mechanisms may account for DCD in preterm compared with fullterm children, which highlights the role of early identification and intervention in this high-risk population. To further examine predictors of DCD, Zwicker is currently determining brain biomarkers for DCD by examining the association of neonatal brain imaging conducted near birth and term-equivalent age to imaging conducted at age 8 in children who were born preterm.

\section{Brain Imaging May Provide Evidence for Rehabilitation Interventions}

Questions about how DCD is explained by brain mechanisms and how the child responds to motor learning and motor skill acquisition is the next frontier in research. Based on principles of motor learning and neuroplasticity, it is conceivable that children with DCD can demonstrate improved motor skill and relatively permanent change in function as a result of motor training $[65,66]$. In the case of dyslexia, for example, increases in gray matter volume were observed in response to reading intervention in dyslexic children after a short 8-week training program [67]. Demonstrating neuroplasticity in response to learning and training has only recently been applied to children with DCD.

Zwicker et al. were the first to track motor training effects using serial MRI scans [22••]. They reported significant differences between groups in a broad network of regions associated with motor learning, including bilateral inferior parietal lobules, right dorsolateral prefrontal cortex, and the cerebellum. The relative under-activation of these regions by children with DCD may be associated with their poor motor learning compared to typically developing peers. It is possible that activation of this network during intervention may induce gains in motor learning in DCD. Future work with a larger sample size is needed to confirm these hypotheses.

Like gray matter, white matter has also been shown to change with development and in association with training and skill acquisition/motor learning [68, 69]. Abnormalities in white matter measured using DTI have been reported in children with DCD. White matter tracts in children with DCD exhibited lower fractional anisotropy and higher diffusion compared to typically developing peers. These findings reflect maturational differences in white matter architecture.
An important next step in advancing our understanding of DCD is to establish typical trajectory of motor pathway development over time using serial scans to inform diagnosis and neural responses to skill development/motor learning. Studies to examine whether neuroplastic changes occur with rehabilitation intervention is currently underway in a clinical trial conducted by Zwicker in Vancouver, Canada.

\section{Future Directions}

Although neuroimaging studies have greatly informed our understanding of DCD, most studies are limited by small sample sizes and thus need to be replicated to confirm findings. In addition, studies have used different cutoff scores to define DCD, but all have found significant brain differences compared to typically developing children. These findings partially confirm the EACD guidelines to use a more generous cutoff in diagnosing DCD. Neuroimaging studies with larger sample sizes may help determine if there are brain differences in children who score $\leq 5$ th percentile compared with children who score $\leq 16$ th percentile on the MABC-2. Further work in determining the neural correlates of DCD may ultimately inform diagnosis of DCD in the long term. Because most of the neuroimaging findings in DCD are related to differences in brain activation or are at the microstructure level, using MRI for diagnosis is not practical at this time; however, advances in MR technology and increased accessibility to MRI may make this a possibility in the future with continued efforts to determine neural correlates of DCD.

\section{Conclusion}

Advanced imaging techniques have provided a window into the neural correlates of DCD, delivering essential information on brain structure and function. Together, these findings inform the neural basis of DCD and will help to confirm or develop interventions for children with DCD that will optimize neurodevelopmental outcomes. These findings are the tip of the iceberg, as questions remain regarding the optimal application of this knowledge of neural correlates to diagnosis and intervention.

Acknowledgments Dr. Zwicker is the recipient of a Michael Smith Foundation for Health Research Scholar Award and a Canadian Child Health Clinician Scientist Program Career Enhancement Award.

\section{Compliance with Ethics Guidelines}

Conflict of Interest Meisan Brown-Lum and Jill Zwicker declare that they have no conflict of interest.

Human and Animal Rights and Informed Consent This article does not contain any studies with human or animal subjects performed by any of the authors. 


\section{References}

Papers of particular interest, published recently, have been highlighted as:

- Of importance

- Of major importance

1. American Psychiatric Association. Diagnostic and Statistical Manual of Mental Disorders (DSM-5). Author: Washington, DC. 2013.

2. Kirby A, Sugden D, Purcell C. Diagnosing developmental coordination disorders. Arch Dis Child. 2014;99(3):292-6.

3. Zwicker J, Missiuna C, Harris S, Boyd L. Developmental coordination disorder: a review and update. Eur J Paediatr Neurol. 2012;16(6):573-81.

4. Zwicker J, Harris S, Klassen A. Quality of life domains affected in children with developmental coordination disorder: a systematic review. Child Care Health Dev. 2013;39(4):562-80.

5. Wilson P, Ruddock S, Smits-Engelsman B, Polatajko H, Blank R. Understanding performance deficits in developmental coordination disorder: a meta-analysis of recent research. Dev Med Child Neurol. 2013;55:217-28

This provides an up to date summary of key behavioral studies that have informed the DCD literature.

6. Zwicker J, Missiuna C, Boyd L. Neural correlates of developmental coordination disorder: a review of hypotheses. J Child Neurol. 2009;24(10):1273-81.

7. Peters L, Maathuis C, Hadders-Algra M. Neural correlates of developmental coordination disorder. Dev Med Child Neurol. 2013;55(s4):59-64.

8. Gebauer D, Fink A, Filippini N. Differences in integrity of white matter and changes with training in spelling impaired children: a diffusion tensor imaging study. Brain Struct Funct. 2012;217(3): 747-60.

9. Keller TA, Just M. Altering cortical connectivity: remediationinduced changes in the white matter of poor readers. Neuron. 2009;64(5):624-31.

10. Blank R, Smits-Engelsman B, Polatajko H, Wilson P. Recommendations of the definition, diagnosis and intervention of developmental coordination disorder (long version). Dev Med Child Neurol. 2012;54:54-93.

11. Henderson S, Sugden D, Barnett A. Movement assessment battery for children - 2 Examiner's manual. London: Harcourt assessment, 2007. Examiner's Manual. London: Harcourt Assessment. 2007.

12. Mattay V, Weinberger D. Organization of the human motor system as studied by functional magnetic resonance imaging. Eur J Radiol. 1999;30(2):105114.

13. Wilmut K, Brown JH, Wann JP. Attention disengagement in children with developmental coordination disorder. Disabil Rehabil. 2007;29(1):47-55.

14. Groenewegen HJ. The basal ganglia and motor control. Neural Plast. 2003;10(1-2):107-20.

15. Ivry R. Cerebellar involvement in clumsiness and other developmental disorders. Neural Plast. 2003;10(1-2):141-53.

16. Visser J. Developmental coordination disorder: a review of research on subtypes and comorbidities. Hum Mov Sci. 2003;22(4-5):47993.

17. Wilson PH, Maruff $\mathrm{P}, \mathrm{McKenzie} \mathrm{BE}$. Covert orienting of visuospatial attention in children with developmental coordination disorder. Dev Med Child Neurol. 1997;39(11):736-45.

18.• Querne L, Berquin P, Vernier-Hauvette M-P, Fall S, Deltour L, Meyer M-E, et al. Dysfunction of the attentional brain network in children with developmental coordination disorder: a fMRI study. Brain Res. 2008;1244:89-102
This paper is significant as it was the first to use fMRI to investigate attentional brain network in children with DCD.

19. Debrabant J, Gheysen F, Caeyenberghs K, Waelvelde H, Vingerhoets G. Neural underpinnings of impaired predictive motor timing in children with developmental coordination disorder. Res Dev Disabil. 2013;34(5):1478-87.

20. Iwaki S, Kashiwagi M, Narumi Y, Tamai H, Suzuki S. Parietal dysfunction in developmental coordination disorder: a functional MRI study. NeuroReport. 2009;20(15):1319.

21. Zwicker J, Missiuna C, Harris S, Boyd L. Brain activation of children with developmental coordination disorder is different than peers. Pediatrics. 2010;126(3):e678-86.

22.• Zwicker J, Missiuna C, Harris S, Boyd L. Brain activation associated with motor skill practice in children with developmental coordination disorder: an fMRI study. Int J Dev Neurosci. 2011;29(2): $145-52$.

This paper is of significant importance as is it one of the few core neuroimaging studies investigating developmental coordination disorder and the first to report motor training effect using serial MRI scans.

23. Adams IL, Lust JM, Wilson PH, Steenbergen B. Compromised motor control in children with DCD: a deficit in the internal model? - a systemaic review. Neurosci Biobehav Rev. 2014. doi:10. 1016/j.neubiorev.2014.08.011.

24. Biswal B, Yetkin ZF. Functional connectivity in the motor cortex of resting human brain using echo-planar mri. Magn Reson Med. 1995;34(4):537-41.

25. Pinotsis DA, Hansen E, Friston KJ, Jirsa VK. Anatomical connectivity and the resting state activity of large cortical networks. NeuroImage. 2013;65:127-38.

26. Cohen AL, Fair DA, Dosenbach N, Miezin FM. Defining functional areas in individual human brains using resting functional connectivity MRI. NeuroImage. 2008;41:45-57.

27. Lin W, Zhu Q, Gao W, Chen Y, Toh CH. Functional connectivity MR imaging reveals cortical functional connectivity in the developing brain. Am J Neuroradiol. 2008;29:1883-9.

28. Wang L, Zhu C, He Y, Zang Y, Cao QJ. Altered small-world brain functional networks in children with attention-deficit/hyperactivity disorder. Hum Brain Mapp. 2009;30:638-49.

29. Martino DA, Kelly C, Grzadzinski R, Zuo XN. Aberrant striatal functional connectivity in children with autism. Biol Psychiatry. 2011;69:847-56.

30. McLeod KR, Langevin LM, Goodyear BG, Dewey D. Functional connectivity of neural motor networks is disrupted in children with developmental coordination disorder and attention-deficit/hyperactivity disorder. NeuroImage Clin. 2014;4:566-75.

31. Niedermeyer E, da Silva F. Electroencephalography: basic principles, clinical applications, and related fields. Philadelphia: Lippincott Williams \& Wilkins; 2005.

32. Castelnau P, Albaret J-M, Chaix Y, Zanone P-G. A study of EEG coherence in DCD children during motor synchronization task. Hum Mov Sci. 2008;27(2):230-41.

33. Albaret J-M, Chaix Y. Neurobiological bases and neurophysiological correlates of developmental coordination disorders. Clin Neurophysiol. 2011;42(1-2):11-7

This paper is one of the more recent EEG studies to investigate cortical activation patterns in children with DCD.

34. Tsai C-L, Pan C-Y, Cherng R-J, Hsu Y-W, Chiu H-H. Mechanisms of deficit of visuospatial attention shift in children with developmental coordination disorder: a neurophysiological measure of the endogenous Posner paradigm. Brain Cogn. 2009;71(3):246-58.

35. Tsai C, Chang Y, Hung T, Tseng Y, Chen T. The neurophysiological performance of visuospatial working memory in children with developmental coordination disorder. Dev Med Child Neurol. 2012;54(12):1114-20

Being one of the more recent EEG studies, this paper is 
important for its contribution to the visuospatial working memory hypothesis in children with DCD.

36. Pangelinan M, Hatfield B, Clark J. Differences in movement-related cortical activation patterns underlying motor performance in children with and without developmental coordination disorder. J Neurophysiol. 2013;109(12):3041-50

This study was unique to look at motor performance and cortical activation of typically- developing children and children with DCD across different developmental age groups.

37. Beaulieu C. The basis of anisotropic water diffusion in the nervous system - a technical review. NMR Biomed. 2002;15:435-55.

38. Snook L, Paulson LA, Roy D, Phillips L, Beaulieu C. Diffusion tensor imaging of neurodevelopment in children and young adults. NeuroImage. 2005;26:1164-73.

39.• Zwicker J, Missiuna C, Harris S, Boyd L. Developmental coordination disorder: a pilot diffusion tensor imaging study. Pediatr Neurol. 2012;46(3):162-7

This paper is the first to characterize white matter microstructure in children with DCD and typically-developing children and to correlate that with motor outcomes. This paper was recognized by the American Academy of Cerebral Palsy and Developmental Medicine as one of the top ten publications in childhood disability published in $\mathbf{2 0 1 2}$

40.• Langevin L, MacMaster F, Crawford S, Lebel C, Dewey D. Common white matter microstructure alterations in pediatric motor and attention disorders. J Pediatr. 2014;164(5):1157-1164.e1

This paper is only one of two neuroimaging studies that characterizes white matter microstructure in children with DCD.

41. Kaplan J, Wilson BN, Dewey D, Crawford SG. DCD may not be a discrete disorder. Hum Mov Sci. 1998;17:471-90.

42. Gilger JW, Kaplan BJ. Atypical brain development: a conceptual framework for understanding developmental learning disabilities. Dev Neuropsychol. 2001;20(2):465-81.

43. Wilson $\mathrm{PH}, \mathrm{McKenzie} \mathrm{BE}$. Information processing deficits associated with developmental coordination disorder: a meta-analysis of research findings. J Child Psychol Psychiatry. 1998;39(6):829-40.

44. Zatorre RJ, Fields RD, Johansen-Berg H. Plasticity in gray and white: neuroimaging changes in brain structure during learning. Nat Neurosci. 2012;15(4):528-36.

45.• Langevin L, MacMaster F, Dewey D. Distinct patterns of cortical thinning in concurrent motor and attention disorders. Dev Med Child Neurol. 2014. doi:10.1111/dmcn.12561

This paper is the first study to characterize cortical thinning in children with DCD.

46. Mariën P, Wackenier P, Surgeloose D, Deyn P, Verhoeven J. Developmental coordination disorder: disruption of the cerebellocerebral network evidenced by SPECT. Cerebellum. 2010;9(3): 405410.

47. Cantin N, Polatajko HJ, Thach WT, Jaglal S. Developmental coordination disorder: exploration of a cerebellar hypothesis. Hum Mov Sci. 2007;26:491-509.

48. Fawcett AJ, Nicolson RI. Automatisation deficits in balance for dyslexic children. Percept Mot Skills. 1992;75:507-29.

49. Nicolson RI, Fawcett AJ, Dean P. Time estimation deficits in developmental dyslexia: evidence of cerebellar involvement. Proc R Soc Lond B. 1995;259:43-7.

50. Ito M. Mechanisms of motor learning in the cerebellum. Brain Res. 2000;886:237-45.

51. Wilson PH, Maruff P, Buston M, Williams J, Lum J, Thomas PR. Internal representation of movement in children with developmental coordination disorder: a mental rotation task. Dev Med Child Neurol. 2004;46:754-9.

52. Blakemore SJ, Sirigu A. Action prediction in the cerebellum and in the parietal lobe. Exp Brain Res. 2003;153:239-45.

53. Lust J, Geuze R, Wijers A, Wilson P. An EEG study of mental rotation-related negativity in children with developmental coordination disorder. Child Care Health Dev. 2006;32(6):649-63.

54. Wilson PH, Maruff P, Ives S, Currie J. Abnormalities of motor and praxis imagery in children with DCD. Hum Mov Sci. 2001;20:13559.

55. Maruff P, Wilson P, Trebilcock M, Currie J. Abnormalities of imaged motor sequences in children with developmental coordination disorder. Neuropsychologia. 1999;37:1317-24.

56. Sirigu A, Duhamel JR, Cohen L, Pillon B, Dubois B, Agid Y. The mental representation of hand movements after parietal cortex damage. Science. 1996;273:1564-8.

57. Edwards J, Berube M, Erlandson K. Developmental coordination disorder in school-aged children born very preterm and/or at very low birth weight: a systematic review. J Dev Behav Pediatr. 2011;32(9):678-87.

58. Spittle AJ, Cheong J, Doyle LW. Neonatal white matter abnormality predicts childhood motor impairment in very preterm children. Dev Med Child Neurol. 2011;53:1000-6

In this paper, white matter injury has been hypothesized to account for motor impairment in children born preterm.

59. Abernethy LJ, Cooke R, Foulder-Hughes L. Caudate and hippocampal volumes, intelligence, and motor impairment in 7-year-old children who were born preterm. Pediatr Res. 2004;55(5):884-93.

60. Tiemeier H, Lenroot R, Greenstein D, Tran L, Pierson R, Giedd J. Cerebellum development during childhood and adolescence: a longitudinal morphometric MRI study. NeuroImage. 2010;49(1):6370 .

61. Rademaker KJ, Lam J, Haastert VI. Larger corpus callosum size with better motor performance in prematurely born children. Semin Perinatol. 2004;28(4):279-87.

62. De Kieviet JF, Pouwels P, Lafeber HN. A crucial role of altered fractional anisotropy in motor problems of very preterm children. Eur J Paediatr Neurol. 2014;18:126-33

This paper reports on the relationship between white matter microstructure and motor impairment and DCD in very preterm children at school age.

63. Zwicker J. Motor impairment in very preterm infants: implications for clinical practice and research. Dev Med Child Neurol. 2014;56(6):514-5.

64. Zwicker J, Yoon S, Mackay M, Petrie-Thomas J, Rogers M, Synnes A. Perinatal and neonatal predictors of developmental coordination disorder in very low birthweight children. Arch Dis Child. 2013;98(2):118-22.

65. Schmidt RA, Lee T. Motor control and learning. Champaign: Human Kinetics; 2005.

66. Kleim JA, Jones TA. Principles of experience-dependent neural plasticity: implications for rehabilitation after brain damage. J Speech Lang Hear Res. 2008;51(1):S225-39.

67. Krafnick AJ, Flowers DL, Napoliello EM, Eden GF. Gray matter volume changes following reading intervention in dyslexic children. NeuroImage. 2011;57:733-41.

68. Scholz J, Klein MC, Behrens T. Training induces changes in whitematter architecture. Nat Neurosci. 2009;12(11):1370-1.

69. Johansen-Berg H. Structural plasticity: rewiring the brain. Curr Biol. 2007;17(4):R141-4. 